\title{
EFEITO DA ADIÇÃO DE EMULSIFICANTES EM MASSAS ALIMENTICIAS SEM GLÚTEN PRODUZIDAS COM EXTRUSORA TERMOPLÁSTICA
}

\author{
ELIZABETH HARUMI NABESHIMA * \\ JORGE MINORU HASHIMOTO ** \\ AHMED ATIA EL-DASH ***
}

\begin{abstract}
Foram produzidas massas alimentícias instantâneas sem glúten a partir de farinha de arroz suplementadas com aditivos, utilizando-se extrusor de laboratório Brabender de rosca única. Foi testada a adição dos emulsificantes, estearoil lactil lactato de sódio (Esterlac) e monoglicerídio destilado (Dimodan), ambos nas concentrações de 0,$00 ; 0,25 ; 0,50,0,75$ e 1,00\%, além da mistura dos mesmos $(0,00: 1,00 ; 0,50: 0,50 ; 0,75: 0,25$ e $0,00: 1,00 \%)$. Também foram estudadas as propriedades de cozimento. A adição de emulsificante tanto individualmente como em mistura melhorou a qualidade do produto, principalmente pela propriedade lubrificante. Essa facilitou o processo de extrusão e possibilitou o uso de temperaturas de extrusão acima de $90^{\circ} \mathrm{C}$ sem formação de bolhas no interior das massas. O uso do Dimodan exerceu maior influência sobre o produto do que o Esterlac, principalmente em relação ao aumento de volume e de peso do produto cozido. Os melhores resultados foram obtidos com a mistura de $0,75 \%$ de Dimodan e $0,25 \%$ de Esterlac. No entanto, as massas alimentícias contendo Dimodan, individualmente ou em misturas, apresentaram rachaduras laterais após o cozimento.
\end{abstract}

PALAVRAS-CHAVE: MASSAS ALIMENTÍCIAS, GLÚTEN, EMULSIFICANTES, FARINHA DE ARROZ; EXTRUSÃO TERMOPLÁSTICA.

* Doutoranda, Bolsista CNPq, Departamento de Tecnologia de Alimentos (DTA), Faculdade de Engenharia de Alimentos (FEA), Universidade Estadual de Campinas (UNICAMP), Campinas, SP (e-mail: ehnabeshima@hotmail.com).

** Doutorando, Departamento de Ciências de Alimentos (DCA), FEA, UNICAMP, Campinas, SP (e-mail: jorgehashimoto@yahoo.com.br).

*** Professor titular, DTA, FEA, UNICAMP, Campinas, SP

(e-mail: ahmed@fea.unicamp.br). 


\section{INTRODUÇÃO}

Os emulsificantes são importantes aditivos utilizados no preparo de massas alimentícias, atuando na redução da perda de sólidos na água de cozimento e na melhoria da textura (adesividade e firmeza). Além disso, aumentam a uniformidade, a espessura, o brilho e a dureza das massas (PAPE e CAMPOS, 1971; KOVÁCS et al., 1992; KAMEL, 1997; PAVANELLI et al, 1999). De acordo com KAMEL (1997) a adição de emulsificantes também proporciona melhor controle do processo, devido à propriedade de lubrificação dos produtos amiláceos extrusados que facilita a passagem da massa através da matriz.

Os principais emulsificantes utilizados pela indústria alimentícia são os monoglicerídios e os ésteres de ácidos lácticos, os quais são selecionados de acordo com as suas propriedades. Os monoglicerídios, mais comumente empregados, pertencem à categoria dos produtos geralmente reconhecidos como seguros (GRAS). São permitidos em concentrações não-limitadas nas legislações internacionais (KAMEL, 1997), enquanto que a legislação brasileira limita a concentração em 0,5 g/100 g de farinha para massas alimentícias (BRASIL, 2002a). Podem ser adicionados em quantidades maiores, desde que as necessidades tecnológicas e as técnicas de boas práticas de fabricação justifiquem a concentração utilizada (BRASIL, 2002b).

Os ésteres de ácido láctico são produtos da reação do ácido esteárico (50-90\%, C:18) com o ácido láctico. Podem ser divididos em dois grupos, os ésteres iônicos e os não-iônicos. Os não-iônicos são amplamente utilizados na panificação e como cobertura, mas não formam fases mesomórficas com a água. Os iônicos também são utilizados em produtos de panificação, sendo os mais importantes o estearoil lactil lactato de cálcio e o estearoil lactil lactato de sódio. São permitidos tanto nos Estados Unidos (KAMEL, 1997), como no Brasil na concentração de $0,5 \mathrm{~g} / 100 \mathrm{~g}$ de farinha (BRASIL, 2002a).

Conforme KOVÁCS et al. (1992) e KAMEL (1997) não há ordem específica de adição dos emulsificantes, no entanto deve ser executada de forma a garantir a homogeneização da mistura.

PAPE e CAMPOS (1971) estudaram o comportamento do estearoil lactil-lactato de cálcio (ELLC) e do estearoil lactil-lactato de sódio 
(ELLS) na fabricação de massas alimentícias. Utilizaram semolina pura ou farinha de trigo com ou sem amido de milho para elaborar espaguete e talharim, adicionados de ovos e/ou emulsificantes. Observaram que os produtos com adição de ELLS apresentaram qualidade superior aos produtos adicionados de ELLC (melhor sabor, maior espessura, dureza e brilho, e menor perda de sólidos, pegajosidade e empapamento ou formação de goma). No entanto, as variações foram insignificantes quanto ao aumento de peso após o cozimento, sendo que a amostra teste evidenciou absorção de água ligeiramente maior.

ORMENESE (1998) comparou o efeito da adição de monoglicerídios destilados (MGD) e ELLS nas características de cozimento de massas alimentícias de arroz obtidas pelo processo convencional. Verificou que tanto o MGD como o ELLS diminuiram consideravelmente a perda de sólidos solúveis (7,8 e 8,5\% respectivamente, contra 17,1\% para a massa sem aditivo).

Os efeitos dos emulsificantes sobre as massas alimentícias podem ser explicados pela capacidade de complexação com o amido, sendo que o processo de extrusão termoplástica pode promover outros tipos de transformações. SOARES (1995) estudou o efeito da adição de monoglicerídios nas propriedades de amido de mandioca extrusado em extrusor termoplástico de laboratório (Cerealtec) de rosca única. Constatou aumento na dureza dos géis, decorrente da redução da solubilidade em água produzida pela formação do complexo amilosemonoglicerídio. Assim, a fração solúvel em água diminuiu e a proporção de amilopectina em soluções ou suspensões de amido-água aumentou, provocando maior dureza do gel em comparação com extrusados sem monoglicerídios.

ELIASSON (1985) testou o efeito dos emulsificantes dodecil sulfato de sódio (SDS), ELLS e monoestearato de glicerila saturado (GMS) em amido de trigo, utilizando extrusor termoplástico e diferentes temperaturas. $\mathrm{O}$ uso de emulsificante afetou o transporte de água para o interior do grânulo, devido à formação do complexo amidomonoglicerídio. Tais complexos, formados na superfície do grânulo, criam filme insolúvel que retarda o transporte de água para dentro do grânulo e o intumescimento. 
Este trabalho teve como objetivo verificar o efeito dos emulsificantes estearoil lactil lactato de sódio e monoglicerídio destilado no preparo de massas alimentícias instantâneas sem glúten, com base nas propriedades de cozimento.

\section{MATERIAL E MÉTODOS}

\subsection{MATERIAL}

\subsubsection{Matérias-primas}

O arroz (Oriza sativa) variedade Agulha, fornecido pela cerealista Coradine Ltda. (Porto Alegre-RS), foi moído em moinho de rolos Brabender (modelo Quadrumat Senior), e submetido aos processos de quebra e redução para transformação em farinha de arroz.

Foram utilizados Estearoil Lactil Lactato de Sódio (Esterlac $\left.{ }^{\circledR}\right)$, fornecido pela Sínteses Purac (São Paulo-SP) e Monoglicerídio Destilado (Dimodan ${ }^{\circledR}$ ), fornecido pela Rhodia (São Paulo-SP).

\subsection{CARACTERIZAÇÃO DA FARINHA DE ARROZ}

A caracterização físico-química da farinha de arroz consistiu na determinação do teor de umidade, proteínas, lipídios totais e cinzas de acordo com os métodos analíticos da AACC (2000). O teor de carboidratos foi determinado por diferença e o de amilose pela metodologia de BHATTACHARYA et al. (1972).

\subsection{PRODUÇÃO DAS MASSAS ALIMENTÍCIAS PELO PROCESSO DE EXTRUSÃO TERMOPLÁSTICA}

\subsubsection{Preparo das Formulações}

Cada formulação de massa alimentícia instantânea sem glúten foi constituída de $1.000 \mathrm{~g}$, sendo os emulsificantes adicionados nas quantidades apresentadas nas Tabela 1 e 2. Para melhor homogeneização efetuou-se mistura manual com $200 \mathrm{~g}$ de farinha de 
arroz e emulsificante (\% indicada nas Tabelas 1 e 2 ). 0 restante da farinha foi complementado posteriormente e misturado em batedeira planetária (KitchenAid Professional, modelo K45SS) em velocidade baixa, por 10 minutos. Nesse tempo, a mistura foi condicionada para umidade de $23 \%$, definida conforme testes preliminares. A quantidade de água adicionada foi determinada pela seguinte fórmula:

$$
\% \text { água }=\frac{(100-\text { umidade inicial da mistura seca })}{(100-\text { umidade final desejada })} \times \text { peso total da mistura }(\mathrm{g})
$$

As formulações foram acondicionadas em sacos plásticos, permanecendo na geladeira $\left(\mathrm{a} 10^{\circ} \mathrm{C}\right.$ por 12 horas) até o momento da extrusão.

TABELA 1 - TIPO E QUANTIDADE DE EMULSIFICANTES ESTEAROIL LACTIL LACTATO DE SÓDIO (ELLS) E MONOGLICERÍDIO DESTILADO (MGD), UTILIZADOS NO PREPARO DAS MASSAS ALIMENTÍCIAS SEM GLÚTEN

\begin{tabular}{ccc}
\hline Ensaios & \multicolumn{2}{c}{ Tipo Emulsificante } \\
& ELLS $(\%)$ & MGD (\%) \\
\hline 1 (controle) & - & - \\
2 & 0,25 & - \\
3 & 0,50 & - \\
4 & 0,75 & - \\
5 & 1,00 & - \\
6 & - & 0,25 \\
7 & - & 0,50 \\
8 & - & 0,75 \\
9 & - & 1,00 \\
\hline
\end{tabular}

\subsubsection{Extrusão}

Uma mistura de cada ensaio foi extrusada em extrusor de laboratório Brabender de rosca única (modelo 20 D/N - GNF 1014/2), sob condições fixas de processo. Temperatura de $90^{\circ} \mathrm{C}$ nas $1^{\text {a }}$ e $3^{\mathrm{a}}$ zonas, $80{ }^{\circ} \mathrm{C}$ na $2^{\circ}$ zona, velocidade do parafuso de $120 \mathrm{rpm}$, taxa de compressão do parafuso de 1:3, taxa de alimentação de $70 \mathrm{~g} / \mathrm{min}$ e matriz do tipo laminar (5 orifícios de $0,2 \times 0,8 \mathrm{~mm}$ ). Os produtos extrusados foram secos em estufa com circulação de ar, a $50^{\circ} \mathrm{C}$, até 
teor de umidade inferior a $12 \%$. A seguir, foram acondicionados em sacos plásticos e armazenados em temperatura ambiente até a realização das análises.

TABELA 2 - PROPORÇÕES DA MISTURA DE EMULSIFICANTES ESTEAROIL LACTIL LACTATO DE SÓDIO (ELLS) E MONOGLICERÍDIO DESTILADO (MGD) UTILIZADOS NO PREPARO DE MASSAS ALIMENTÍCIAS SEM GLÚTEN

\begin{tabular}{cc}
\hline ENSAIO & ELLS + MGD \\
\hline 1 & $0,50 \%+0,50 \%$ \\
2 & $0,25 \%+0,75 \%$ \\
3 & $0,75 \%+0,25 \%$ \\
4 & $0,00 \%+1,00 \%$ \\
5 & $1,00 \%+0,00 \%$ \\
\hline
\end{tabular}

\subsection{DELINEAMENTO EXPERIMENTAL}

Estudou-se o efeito da adição dos emulsificantes sobre as propriedades das massas alimentícias instantâneas sem glúten, extrusadas em extrusor termoplástico, sob condições fixas, estabelecidas de acordo com a literatura e análises preliminares. As concentrações de emulsificantes utilizadas para o preparo da mistura constam na Tabela 1 e 2.

A significância estatística das diferenças entre as médias foi determinada mediante teste de Tukey, utilizando o programa Statistica 5.0 (STATSOFT, 1995).

\subsection{CARACTERIZAÇÃO TECNOLÓGICA}

O teste de cozimento foi realizado segundo metodologia da AACC 1650 (2000). Para cada ensaio, realizado em triplicata, foram utilizadas $10 \mathrm{~g}$ de produto $(5 \mathrm{~cm})$ cozido em béquer contendo $140 \mathrm{~mL}$ de água destilada fervente. Após atingir o tempo ótimo de cocção, a água de cozimento foi drenada mediante funil de Buchner. 
O tempo ótimo de cozimento foi definido como o tempo requerido para o centro da massa alimentícia tornar-se esbranquiçada indicando que o amido no centro da massa está hidratado. Esse ponto foi estabelecido pela remoção de pedaços de massa alimentícia, em intervalos de 30 segundos, observando-se visualmente a mudança.

O ganho de peso foi determinado pela pesagem de $10 \mathrm{~g}$ de amostra crua e após a cocção (tempo ótimo). Calculou-se a diferença de peso entre a amostra crua e cozida, sendo os resultados expressos em porcentagem.

Determinou-se o volume das amostras, utilizando $50 \mathrm{~mL}$ de solvente (hexano) em proveta de $100 \mathrm{~mL}$. A razão entre o volume de solvente deslocado pelo macarrão cru (10 g) e o cozido serviu como parâmetro para estabelecimento do aumento de volume. Os valores foram expressos em porcentagem (AACC, 2000).

\section{RESULTADOS E DISCUSSÃO}

A composição centesimal da farinha de arroz, utilizada no preparo das massas alimentícias instantâneas, consta da Tabela 3. Essa farinha é classificada como intermediária quanto ao teor de amilose (22-25\%), sendo que quantidades superiores a $22 \%$ são consideradas mais adequadas para o preparo de massas alimentícias sem glúten (KOHLWEY et al., 1995).

\section{TABELA 3 - RESULTADO DA ANÁLISE DE COMPOSIÇÃO CENTESIMAL DA FARINHA DE ARROZ}

\begin{tabular}{lc}
\hline Composi a o Centesimal & \% (Base Seca) \\
\hline Lip dios & 0,63 \\
Prote na $(\mathrm{N} \times 6,25)$ & 7,2 \\
Cinzas & 0,49 \\
Fibra & 0,01 \\
Carboidratos $\quad$ - amilose & 91,67 \\
\end{tabular}


As condições do processo de extrusão (temperatura, umidade e rotação do parafuso) foram ajustadas de modo a evitar a expansão do produto, já que o uso do extrusor termoplástico visava promover a instantaneização e formatação do produto. Conforme COLONNA et al. (1989), a capacidade de expansão dos produtos extrusados pode ser reduzida pela combinação de alguns parâmetros do processo de extrusão. Assim, ressaltaram a diminuição da temperatura e da velocidade do parafuso e o aumento da umidade. Afirmaram que a adição de certos tipos de lipídios (emulsificantes) também inibe ou diminui a expansão, provavelmente por atuarem como lubrificantes e assim promoverem modificações moleculares menos extensivas.

Segundo CHUNGCHAROEN e LUND (1987) a adição de agentes de superfície ativa em sistema amiláceo promove aumento na temperatura de gelatinização. Tal fato ocorre devido à formação do complexo lipídioamilose, que inibe o inchamento e a solubilização do grão de amido.

Neste estudo, as massas alimentícias instantâneas cruas contendo os emulsificantes estearoil lactil lactato de sódio (ELLS) e/ou monoglicerídio destilado (MGD) apresentaram superfície lisa e coloração amarela homogênea. Por outro lado, o produto controle (sem adição de emulsificante) apresentou bolhas de ar (característica de produtos extrusados nas condições empregadas). Observou-se também que os produtos obtidos, diferentemente do que ocorre nos processos convencionais, apresentaram baixa perda por quebra após o acondicionamento em saco plástico de polietileno.

As Tabelas 4 e 5 apresentam os resultados das propriedades de cozimento das massas alimentícias preparadas com a adição de emulsificante (0,00 a 1\%). Para a perda de sólidos (Figura 1), a adição de MGD (Tabela 4) não evidenciou diferença significativa entre os ensaios nas concentrações $(0,25$ a $1,0 \%)$ e condições estudadas, diferindo significativamente do controle com redução de até $71,32 \%$. Para o ELLS (Tabela 5) a maior redução ocorreu com a adição de $0,75 \%$ de emulsificante (65,66\%). HUMMEL (1966) afirmou que a massa de trigo de boa qualidade deve apresentar perda de sólidos solúveis de no máximo $6 \%$. 


\section{TABELA 4 - RESULTADOS DAS ANÁLISES DAS PROPRIEDADES DE COZIMENTO DAS MASSAS ALIMENTÍCIAS SEM GLÚTEN, CONTENDO O EMULSIFICANTE MONOGLICERÍDIO DESTILADO (MGD) EM DIFERENTES PORCENTAGENS}

\begin{tabular}{cccccc}
\hline Ensaio & $\begin{array}{c}\text { MGD \% } \\
(\mathrm{g} / 100 \mathrm{~g})\end{array}$ & $\begin{array}{c}\text { Tempo } \\
\text { Cozimento } \\
(\mathrm{m} \text { in })\end{array}$ & $\begin{array}{c}\text { Aumento } \\
\text { Peso }(\%)\end{array}$ & $\begin{array}{c}\text { Aumento } \\
\text { Volume }(\%)\end{array}$ & $\begin{array}{c}\text { Perda S lidos } \\
(\%)\end{array}$ \\
\hline 1 (Controle) & $0,00 \%$ & 5 & $87,97^{\mathrm{b}}$ & $16,52^{\mathrm{b}}$ & $7,95^{\mathrm{a}}$ \\
2 & $0,25 \%$ & 5 & $112,90^{\mathrm{b}, \mathrm{c}}$ & $10,32^{\mathrm{b}}$ & $2,28^{\mathrm{b}}$ \\
3 & $0,50 \%$ & 5 & $143,35^{\mathrm{a}, \mathrm{c}}$ & $25,30^{\mathrm{a}}$ & $3,61^{\mathrm{b}}$ \\
4 & $0,75 \%$ & 5 & $147,35^{\mathrm{a}, \mathrm{c}}$ & $25,30^{\mathrm{a}}$ & $3,02^{\mathrm{b}}$ \\
5 & $1,00 \%$ & 5 & $173,32^{\mathrm{a}}$ & $30,30^{\mathrm{a}}$ & $3,06^{\mathrm{b}}$ \\
\hline
\end{tabular}

* Médias com letras iguais na mesma coluna não diferem significativamente entre si $(p \leq 0,05)$.

\section{TABELA 5 - RESULTADOS DAS ANÁLISES DAS PROPRIEDADES DE COZIMENTO DAS MASSAS ALIMENTÍCIAS SEM GLÚTEN, CONTENDO EMULSIFICANTE ESTEAROIL LACTIL LACTATO DE SÓDIO (ELLS) EM DIFERENTES PORCENTAGENS}

\begin{tabular}{cccccc}
\hline Ensaio & $\begin{array}{c}\text { ELLS \% } \\
(\mathrm{g} / 100 \mathrm{~g})\end{array}$ & $\begin{array}{c}\text { Tempo } \\
\text { Cozimento } \\
(\mathrm{min})\end{array}$ & $\begin{array}{c}\text { Aumento } \\
\text { Peso }(\%)\end{array}$ & $\begin{array}{c}\text { Aumento } \\
\text { Volume }(\%)\end{array}$ & $\begin{array}{c}\text { Perda } \\
\text { lidos }(\%)\end{array}$ \\
\hline 1 (controle) & $0,00 \%$ & 5 & $87,97^{\mathrm{c}}$ & $16,52^{\mathrm{c}}$ & $7,95^{\mathrm{a}}$ \\
6 & $0,25 \%$ & 5 & $98,65^{\mathrm{b}, \mathrm{c}}$ & $17,65^{\mathrm{b}, \mathrm{c}}$ & $6,95^{\mathrm{a}, \mathrm{b}}$ \\
7 & $0,50 \%$ & 5 & $113,45^{\mathrm{a}}$ & $20,00^{\mathrm{a}, \mathrm{b}}$ & $6,34^{\mathrm{a}, \mathrm{b}}$ \\
8 & $0,75 \%$ & 5 & $108,94^{\mathrm{a}, \mathrm{b}}$ & $19,47^{\mathrm{a}, \mathrm{b}}$ & $5,22^{\mathrm{b}}$ \\
9 & $1,00 \%$ & 5 & $112,42^{\mathrm{a}}$ & $20,35^{\mathrm{a}}$ & $5,64^{\mathrm{a}, \mathrm{b}}$ \\
\hline
\end{tabular}

* Médias com letras iguais na mesma coluna não diferem entre si $(p \leq 0,05)$.

Verificou-se que o aumento de peso (Figura 2) referente ao ensaio 1 (controle) diferiu significativamente dos ensaios 3, 4 e 5 (contendo 0,50, 0,75 e 1,0\%, respectivamente). De acordo com HUMMEL (1966), as massas de boa qualidade devem apresentar aumento de 2 vezes em relação ao peso original. Comparando os ensaios 1 (controle) e 5 verificou-se que a adição do emulsificante MGD resultou em incremento de $97 \%$ no aumento de peso. Tais valores são superiores aos obtidos com o ELLS, cujo maior valor de aumento de peso foi de $24,45 \%$. 
FIGURA 1-EFEITO DA ADIÇÃO DE EMULSIFICANTES ESTEAROIL LACTIL LACTATO DE SÓDIO (ELLS) E MONOGLICERÍDIO DESTILADO (MGD), EM DIFERENTES CONCENTRAÇÕES SOBRE A PERDA DE SÓLIDOS

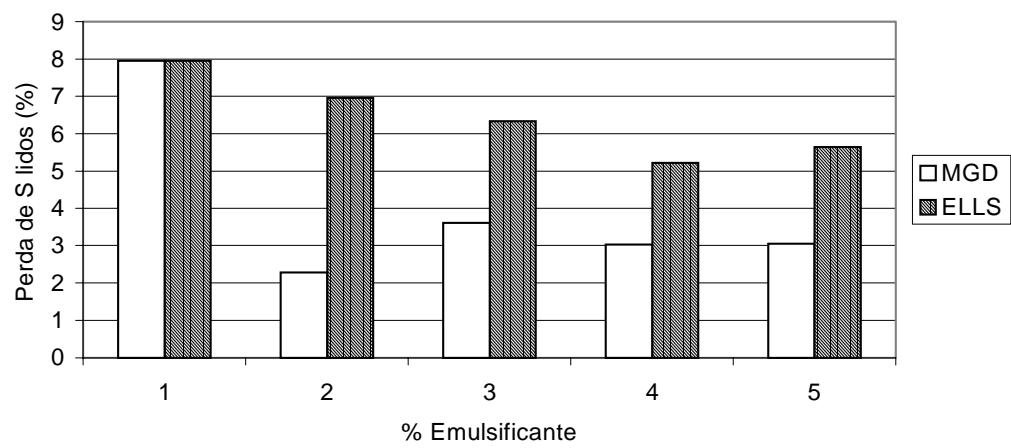

Porcentagens de Emulsificantes: $1=0,00 \% ; \quad 2=0,25 \% ; \quad 3=0,50 \% ; \quad 4=0,75 \%$; $5=1,0 \%$.

FIGURA 2- EFEITO DA ADIÇÃO DE EMULSIFICANTES ESTEAROIL LACTIL LACTATO DE SÓDIO (ELLS) E MONOGLICERÍDIO DESTILADO (MGD), EM DIFERENTES CONCENTRAÇÕESSOBRE O AUMENTO DE PESO

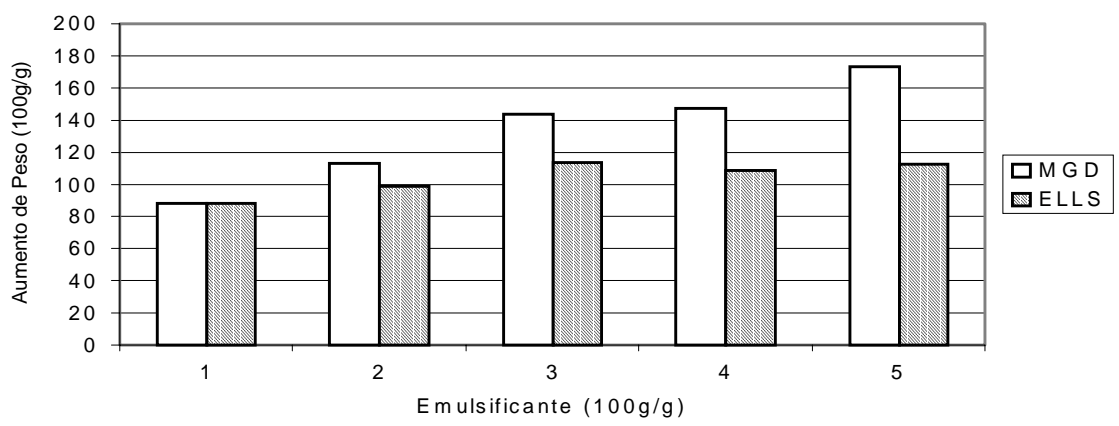

Porcentagens de Emulsificantes: $1=0,00 \% ; \quad 2=0,25 \% ; \quad 3=0,50 \% ; \quad 4=0,75 \%$; $5=1,0 \%$. 
Para o aumento de volume (Figura 3), a adição de 0,25\% de MGD (Tabela 4) não evidenciou diferença em relação controle. Entretanto, diferiu dos ensaios 3, 4 e $5 \mathrm{com}$ aumento de volume de até $83 \% \mathrm{em}$ relação ao controle. Já a adição de ELLS (Tabela 5) resultou em aumento de $23,18 \%$. O aumento desejável para essa propriedade é de 3 a 4 vezes o volume inicial (HUMMEL, 1966).

\section{FIGURA 3-EFEITO DA ADIÇÃO DE EMULSIFICANTES ESTEAROIL LACTIL LACTATO DE SÓDIO (ELLS) E MONOGLICERÍDIO DESTILADO (MGD) EM DIFERENTES CONCENTRAÇÕES SOBRE O AUMENTO DE VOLUME}

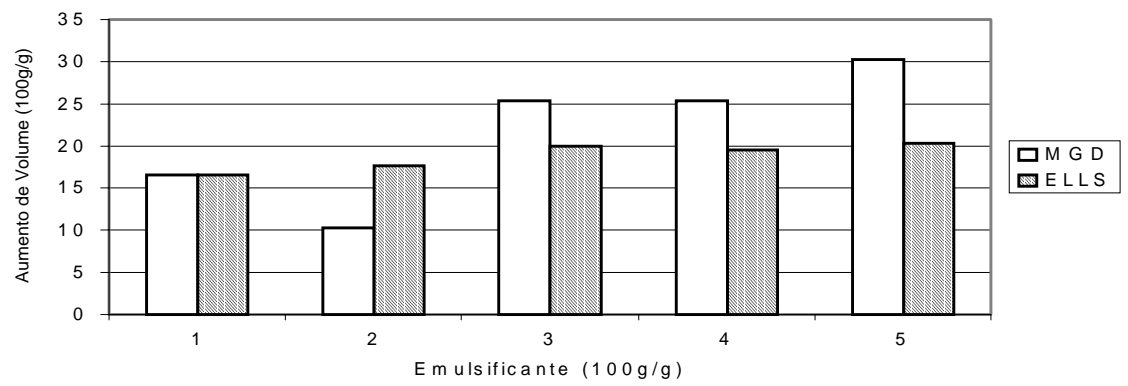

Porcentagens de Emulsificantes: $1=0,00 \% ; 2=0,25 \% ; \quad 3=0,50 \% ; \quad 4=0,75 \%$; $5=1,0 \%$.

Os resultados dos testes de cozimento das massas alimentícias contendo MGD foram comparativamente melhores em relação ao ELLS. Entretanto, as primeiras apresentaram rachaduras laterais após o cozimento. Isso pode ser resultado da retrogradação promovida em maior intensidade na presença do MGD.

Segundo CUNIN et al. (1995) a maior mudança estrutural durante o cozimento das massas ocorre a partir da gelatinização do amido e a coagulação das proteínas. Ambas as transformações ocorrem na mesma temperatura e teor de umidade. $O$ amido gelatinizado contribui para a viscoelasticidade, sendo também identificado como o componente envolvido na adesividade e na desintegração das massas. 
As proteínas são consideradas responsáveis pela boa qualidade das massas cozidas devido à sua habilidade em formar rede insolúvel (glúten) capaz de emaranhar os grânulos de amido gelatinizado e rompidos. Dessa maneira, impedem que a superfície da massa se desfaça durante o cozimento e evitam que o amido seja lixiviado na água de cozimento.

A Tabela 6 apresenta o resultado das propriedades de cozimento das massas alimentícias sem glúten, contendo a mistura dos emulsificantes MGD e ELLS. O aumento da adição de monoglicerídio na mistura proporcionou melhoria na propriedade de cozimento, mas sua adição individual na proporção de $1 \%$ não acompanhou a mesma tendência (Figura 4). O acréscimo da adição de monoglicerídio provocou aumento proporcional das rachaduras laterais das massas após o cozimento.

\section{TABELA 6 - PROPRIEDADES DE COZIMENTO DAS MASSAS ALIMENTÍCIAS DE ARROZ CONTENDO MISTURA DOS EMULSIFICANTES ESTEAROIL LACTIL LACTATO DE SÓDIO (ELLS) E MONOGLICERÍDIO DESTILADO (MGD)}

\begin{tabular}{lccccc}
\hline Ensaio & $\begin{array}{c}\text { MGD + ELLS } \\
(\mathrm{g} / 100 \mathrm{~g} \text { farinha) }\end{array}$ & $\begin{array}{c}\text { Tempo } \\
\text { Cozimento }(\mathrm{min})\end{array}$ & $\begin{array}{c}\text { Aumento } \\
\text { Peso }(\%)\end{array}$ & $\begin{array}{c}\text { Aumento } \\
\text { Volume }(\%)\end{array}$ & $\begin{array}{c}\text { Perda S lidos } \\
(\%)\end{array}$ \\
\hline 1 & $0,00+1,00$ & 5 & $100,38^{\mathrm{d}}$ & $18,18^{\mathrm{c}}$ & $6,00^{\mathrm{a}}$ \\
2 & $0,25+0,75$ & 5 & $139,43^{\mathrm{c}}$ & $25,45^{\mathrm{b}}$ & $4,75^{\mathrm{a}, \mathrm{b}}$ \\
3 & $0,50+0,50$ & 5 & $156,07^{\mathrm{b}}$ & $28,79^{\mathrm{a}}$ & $4,13^{\mathrm{b}}$ \\
4 & $0,75+0,25$ & 5 & $174,22^{\mathrm{a}}$ & $31,45^{\mathrm{a}}$ & $3,07^{\mathrm{c}}$ \\
5 & $1,00+0,00$ & 5 & $129,48^{\mathrm{c}}$ & $22,71^{\mathrm{b}}$ & $3,93^{\mathrm{b}}$ \\
\hline
\end{tabular}

* Médias com letras iguais na mesma coluna não diferem significativamente entre si $(p \leq 0,05)$.

Os maiores valores para os aumentos de peso $(174,22)$ e de volume $(31,45)$, bem como menor perda de sólidos $(3,07)$ foram obtidos com a mistura de 0,75 de Dimodan e $0,25 \%$ de Esterlac (Tabela 6). 
FIGURA 4- EFEITO DA ADIÇÃO DA MISTURA DOS EMULSIFICANTES MONOGLICERÍDIO DESTILADO (MGD) E ESTEAROIL LACTIL LACTATO DE SÓDIO (ELLS) NAS PROPORÇÕES DE 0,00:1,00; 0,25:0,75; 0,50:0,50;0,75:0,25 E 1,0:0,00\% SOBRE A PORCENTAGEM DE PERDA DE SÓLIDOS, AUMENTO DE VOLUME E DE PESO

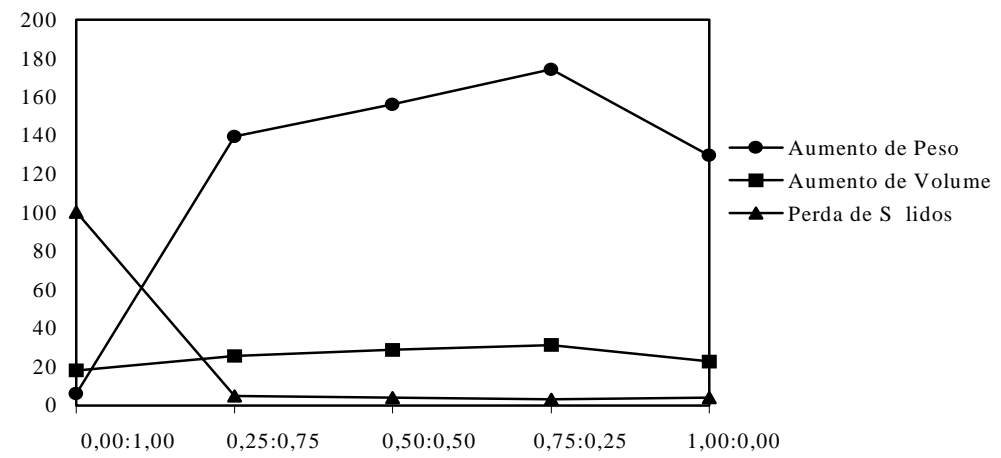

\section{CONCLUSÃO}

A adição de agentes de superfície ativa como o ELLS e MGD, isoladamente ou em misturas, para produção de massas alimentícias instantâneas de farinha de arroz proporcionou benefícios na qualidade do produto final. As massas apresentaram superfície lisa e uniforme, enquanto o controle continha bolhas de ar características de produtos extrusados.

O uso de $0,5 \%$ de MGD ou de ELLS resultou em produtos com maior aumento de peso e de volume em relação ao controle, não havendo diferença significativa entre os produtos contendo maiores quantidades desses emulsificantes $(0,75$ ou $1 \%)$.

As massas com 0,75\% de MGD e 0,25\% de ELLS apresentaram maiores valores de peso e de volume. O MGD exerceu grande influência na qualidade do produto final, comprovado tanto nas combinações 
como no uso isolado de ELLS (que resultou em ganhos menores de qualidade). Apesar do uso de MGD individual ou em mistura proporcionar maior aumento de peso e volume, os produtos apresentaram rachaduras laterais após o cozimento.

Evidenciou-se o potencial de uso de quirera de arroz para a obtenção de produtos com maior valor agregado, de preparo fácil e rápido, que não oferecem maiores dificuldades para o seu armazenamento.

\section{Abstract}

\section{EFFECT OF EMULSIFIERS ADDITION IN PASTA WITHOUT GLUTEN PRODUCED BY THERMOPLASTIC EXTRUDER}

Instantaneous pasta without gluten were produced from rice flour suplemented with additives, prepared by laboratory extruder. The addition of the emulsifiers, sodium stearoyl-2-lactylate (Esterlac ${ }^{\circledR}$ ) and distilled monoglyceride (Dimodan $®$ ) was tested, both in the concentrations of 0,$00 ; 0,25 ; 0,50,0,75$ and $1,00 \%$, and also the mixture $(0,00: 1,00 ; 0,50: 0,50 ; 0,75: 0,25$ and $0,00: 1,00 \%)$. The cooking properties were also studied. The emulsifier addition individually as in mixture brought improvements in the quality of the product, mainly for the lubricating property. This facilitated the extrusion process and made possible the use of temperatures of extrusion over $90^{\circ} \mathrm{C}$, without bubbles formation into the pasta. The use of Dimodan had higher influence on the product than Esterlac, presenting the highest values of volume and weight increase of the cooked product. The best results were obtained through the mixture of $0,75 \%$ Dimodan and $0,25 \%$ Esterlac. However, the pasta containing Dimodan, individually or in mixtures, presented lateral cracks after cooking.

KEY-WORDS: PASTA; GLUTEN; EMULSIFICANTS; RICE FLOUR; THERMOPLASTIC EXTRUSION.

\section{REFERÊNCIAS}

1 AACC. American Association of Cereal Chemists. Approved methods of the AACC. $10^{\text {th }}$ ed. Saint Paul, 2000.

2 BHATTACHARYA, K. R.; SOWBHAGYA, C. M., INDUDHARA SWAMY, Y. M. Interrelatinship between certain physicochemical properties of rice. Journal of Food Science, v.37, p.733-735, 1972.

3 BRASIL. Ministério da Saúde. Secretaria de Vigilância Sanitária. Resolução RDC N³85, de 05 de agosto de 1999. Regulamento técnico que aprova o uso de aditivos alimentares, estabelecendo suas funções e seu limites máximos para a categoria 6: cereais e produtos de ou a base de 
cereais. Disponível em: <http://www.anvisa.gov.br/legis/resol/385_99.htm>. Acesso em 10 dez. 2002.

4 BRASIL. Ministério da Saúde. Secretaria de Vigilância Sanitária. Resolução RDC N³86, de 05 de agosto de 1999. Regulamento técnico sobre aditivos utilizados segundo as boas práticas de fabricação e suas funções. Disponível em: <http://www.anvisa.gov.br/legis/resol/386_99.htm>. Acesso em 10 dez.2002.

5 CHUNGCHAROEN, A.; LUND, D.B. Influence of solutes and water on rice starch gelatinization. Cereal Chemistry, v.64, n.4, p.240-243, 1987.

COLONNA, P.; TAYEB, J.; MERCIER, C. Extrusion cooking for starch and starchy products. In: MERCIER, C.; LINKO, P.; HARPER, J.M. (Eds.). Extrusion technology. Saint Paul: AACC, 1989. Ch.9, p.247-319.

7 CUNIN, C.; HANDSCHIN, S.; WALTHER, P.; ESCHER, F. Structural changes of starch during cooking of durum wheat pasta. Lebens.-Wiss. U.-Technol., v.28, p.323-328, 1995.

8 ELIASSON, A. C. Starch gelatinization in presence of emulsifiers: a morphological study of wheat starch. Starch, v.37, n.12, p.441-451, 1985.

9 HUMMEL, C. Macaroni products. London: Food Trade Press, 1966. 287 p.

10 KAMEL, B.S. Emulsifiers. In: SMITH, J. (Ed.). Foods additive user's handbook. London: Blackie, 1997. Ch.8, p. 169-201.

11 KOHLWEY, D.E.; KENDALL, J.H.; MOHINDRA, R.B. Using the physical properties of rice as a guide to formulation. Cereal Foods World, v.40, n.10, n.728-732, 1995.

12 KOVÁCS, E.; VÁMOS-KARDOS, E.; KISS-LASZLAVIK, M.; PALLAGI, E. Effect of emulsifiers on the properties of pasta products. Acta Alimentaria, v.21, n.3-4, p.205-217, 1992.

13 ORMENESE, R.C.S.C. Influência da adição de emulsificantes nas características de cozimento do macarrão de arroz obtido pelo processo convencional de produção de massas alimentícias. In: CONGRESSO BRASILEIRO DE CIÊNCIA E TECNOLOGIA DE ALIMENTOS - CBCTA, Poços de Caldas, 1998. Anais... Poços de Caldas, 1998. v.2, p.745-757.

14 PAPE, G.; CAMPOS, J.E. Estudo sobre o comportamento de estearoil-lactillactato de cálcio e do estearoil-lactil-lactato de sódio na fabricação de massas alimentícias. Boletim Técnico da Divisão de Tecnologia Agrícola e Alimentar, n.6, p.1-8, 1971.

15 PAVANELLI, A.P.; CICHELLO, M. S.; PALMA, E. P. Emulsificantes como agentes de aeração em bolos. Caderno de Tecnologia de Alimentos e Bebidas, n.2, p.34-38,1999. 
16 SOARES Jr., M.S. Propriedades funcionais de extrusados de amido de mandioca (Manihot esculenta Crantz) com monoglicerídio. Londrina, 1995. 120 p. Dissertação (Mestrado em Ciência de Alimentos), Universidade Estadual de Londrina.

17 STATSOFT. Statistica $\mathbf{5 . 0}$ for windows. Tulsa, OK, USA, 1995. 